\title{
Enzymatic acylation: assessing the greenness of different acyl donors
}

\author{
Monica Paravidino* and Ulf Hanefeld \\ Received 19th May 2011, Accepted 27th June 2011 \\ DOI: 10.1039/c1gc15576h
}

The hydrolase-catalyzed esterification of alcohols is the best established enzymatic transformation in today's organic chemistry, along with the corresponding ester hydrolysis. Over the years, various different acyl donors have been proposed to overcome the major limitation of the condensation of an alcohol and an acid, the unfavourable equilibrium. This review aims at screening the actual number of applications of the different acyl donors, and at assessing the "greenness" (or lack thereof) of the most applied among them. Indeed, the use of an enzyme to catalyze an esterification is often regarded as sufficient to define the whole transformation as "green". However, this definition can easily be misinterpreted if the contribution of the acyl donor to the overall process is overlooked, as is often the case. Aiming at filling this gap, this contribution evaluates the advantages and disadvantages of the acyl donors, and assesses their green credentials using an efficient tool in strategic planning, a strengths-weaknessesopportunities-threats (SWOT) analysis. A calculation of the atom economy and E-factor for representative acylations involving each donor is included, as well as an analysis of the adherence of each process to the twelve principles of Green Chemistry.

\section{What is green about biocatalysis?}

The use of biocatalysis in organic chemistry has come a long way, from academic curiosity a century ago to a standard practice even on an industrial scale today., ${ }^{1,2}$ One reason for this success is the recent trend towards Green Chemistry, ${ }^{3-6}$ i.e. the need for more environmentally acceptable chemical processes. Indeed, biocatalysis performs well in the context of Green Chemistry, offering an environmentally benign catalyst (the enzyme), mild conditions and selectivity at different levels (chemo-, regio- and stereo-). As a result, enzymatic routes are often more attractive than the conventional counterparts from the environmental and economic standpoint. ${ }^{6}$ It is therefore no surprise that a significant number of publications describe enzymatic reactions as being "green". However, the definition of every biocatalytic route as "green" per se is misleading. The use of an enzyme to catalyze a reaction is certainly a good starting point towards a more sustainable chemistry, but does not ensure the greenness of the whole process. Indeed, all aspects (amount and hazardousness of solvents, reagents and waste) have to be carefully taken into account.

Hydrolases $^{2,7,8}$ are a particularly well established class of enzymes and the reactions they catalyze (hydrolysis and formation of esters and amides) are often referred to as green and sustainable. However, while this is reasonably true for the

Gebouw voor Scheikunde, Technische Universiteit Delft, Julianalaan, 136, 2628, BL, Delft, The Netherlands.

E-mail:m.paravidino@tudelft.nl,u.hanefeld@tudelft.nl; Fax: +31 15278 1415; Tel: +31 152785879 hydrolysis, which utilises water as reagent/solvent, the same definition does not apply automatically to the ester and amide synthesis, which requires a suitable acyl donor ${ }^{9-11}$ and often an organic solvent. Indeed, the hydrolase-catalyzed formation of an ester from an alcohol and an acid (transesterification) is a reversible reaction. It is common practice to circumvent this problem, and drive the reaction to completion, by using activated acyl donors rather than acids. The leaving group $\mathrm{X}$ is in this case a weak nucleophile that cannot attack the ester formed (Scheme 1). Thus, a quantitative acylation of the alcohol is ensured.

Although important for determining the sustainability of the esterification, the impact of the acyl donor is usually neglected. With the aim to fill this gap, this contribution offers a guide to the choice of the most suitable acyl donors for esterification reactions for both the laboratory and industrial scale. The synthesis and applications of these acyl donors have already been described in some excellent reviews, ${ }^{9-11}$ to which the reader is referred. Here, the greenness of the most used of them (Table 1) is assessed in comparison with the baseline case, i.e. the condensation of an alcohol and an acid (Scheme 1, X=OH). In addition, the relative advantages and disadvantages of each reagent are evaluated. The green credentials of the acyl donors are evaluated against the relevant among the twelve principles ${ }^{3,4}$ of Green Chemistry, elegantly formulated by Anastas and Warner (Fig. 1). In particular, the $1 \mathrm{st}, 2 \mathrm{nd}, 3 \mathrm{rd}, 10 \mathrm{th}$, and $12 \mathrm{th}$ principles apply to the use of acyl donors as reactant in an enzymatic esterification, and are therefore employed for the discussion here. Moreover, the life cycle assessment (LCA) for the different donors is 


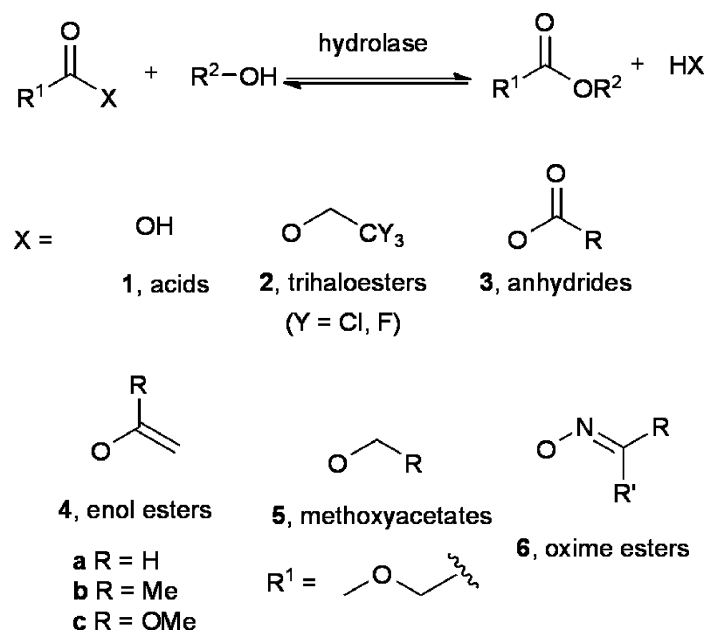

Scheme 1 Illustrates the main acyl donors described in literature.

\section{Twelve Principles of Green Chemistry}

1. It is better to prevent waste than to treat or clean up waste after it has been created.

2. Synthetic methods should be designed to maximise the incorporation of all materials used in the process into the final product.

3. Wherever practicable, synthetic methods should be designed to use and generate substances that possess little or no toxicity to human health and the environment.

4. Chemical products should be designed to preserve efficacy of function while reducing toxicity.

5. The use of auxiliary substances (e.g., solvents, separation agents, etc.) should be made unnecessary wherever possible and innocuous when used.

6. Energy requirements of chemical processes should be recognized for their environmental and economic impacts and should be minimised. If possible, synthetic methods should be conducted at ambient temperature and pressure.

7. A raw material or feedstock should be renewable rather than depleting whenever technically and economically practicable.

8. Unnecessary derivatisation (use of blocking groups, protection/ deprotection, temporary modification of physical/chemical processes) should be minimised or avoided if possible.

9. Catalytic reagents (as selective as possible) are superior to stoichiometric reagents.

10. Chemical products should be designed so that at the end of their function they break down into innocuous degradation products and do not persist in the environment.

11. Analytical methodologies need to be further developed to allow for real-time, inprocess monitoring and control prior to the formation of hazardous substances.

12. Substances and the form of a substance used in a chemical process should be chosen to minimise the potential for chemical accidents, including releases, explosions, and fires.

Fig. 1 The twelve principles of Green Chemistry.

Table 1 Number of applications for each acyl donor

\begin{tabular}{lll}
\hline Entry & Acyl donor & Number of applications \\
\hline 1 & $\mathbf{1}$ & 4 \\
2 & $\mathbf{2}$ & 2 \\
3 & $\mathbf{3}$ & 22 \\
4 & $\mathbf{4 a}$ & 152 \\
5 & $\mathbf{4 b}$ & 9 \\
6 & $\mathbf{4 c}$ & 1 \\
7 & $\mathbf{5}$ & 7 \\
8 & $\mathbf{6}$ & 1
\end{tabular}

Data referred to the time-span 2007-present. Sources: Web of Science and Sci-Finder.

reported whenever available, and additional criteria, viz. atom economy ${ }^{12}$ and E-factor, ${ }^{13}$ are introduced.

Atom economy (or efficiency, eqn (1)) measures how many of the atoms of the starting material end up in the desired product, and is reported as a percentage. The higher the value, the better the economy. However, it should be noted that this parameter does not allow for the effective yield of a reaction nor the need for an excess of reagents, but rather assumes $100 \%$ yield, and is based solely on the stoichiometric equation.

$\%$ Atom economy $=\frac{\text { Molecular weight of desired product }}{\text { Sum of molecular weight of all reagents }} \times 100$

A clearer picture of the greenness of a process can be obtained by calculating its E-factor (eqn (2)), which defines all the compounds that are not product as waste. A truly green reaction has an E-factor close to 0, in accordance to the 2 nd principle of Green Chemistry. ${ }^{3}$

$$
\text { E-factor }=\frac{\text { Amount of waste produced without water }(\mathrm{kg})}{\text { Amount of desired product }(\mathrm{kg})}
$$

Finally, a strengths-weaknesses-opportunities-threats $(\mathrm{SWOT})^{14}$ analysis is presented for each case. This tool provides an overview of the relative advantages and disadvantages of acyl donors and indicates potential directions for improving the process.

\section{Baseline: carboxylic acids}

The enzymatic condensation of an alcohol and an acid needs to be performed under continuous removal of the water, in order to shift the equilibrium towards the product. On a laboratory scale, this protocol (which requires obviously high temperatures) is scarcely applied, and the preference is given to the use of activated acyl donors generally under milder conditions. However, the use of carboxylic acids as acylating agents in enzymatic reactions find some very interesting applications on a large scale. One such example (Scheme 2) ${ }^{15}$ is the synthesis of polyester in preparative scale using glycerol and adipic acid. A feasibility study for the industrial production of this polymer showed that this enzymatic polymerization can be successfully performed at $60{ }^{\circ} \mathrm{C}$ and 20 mbar with only $3 \mathrm{wt} \%$ of Cal-B (Novozym 435). Conversions above $90 \%$ are obtained with a space time yield of $370 \mathrm{~g} \mathrm{~d}^{-1} \mathrm{~L}^{-1}$, which results in an extremely low E-factor (0.35). Moreover, the atom economy is extremely high $(97 \%)$, as expected for a condensation leading to water as the only byproduct.

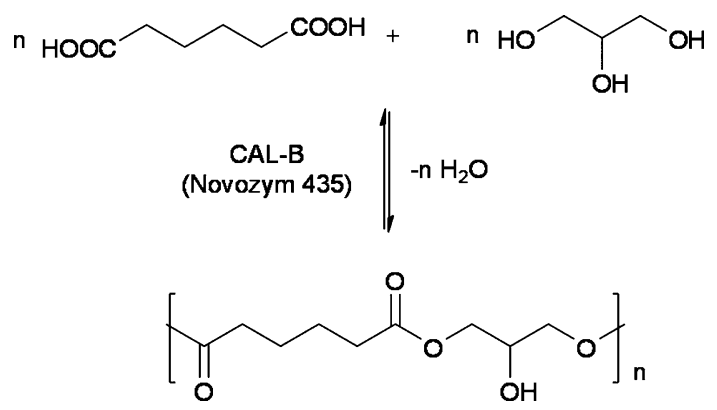

Scheme 2

The applicability of acids at industrial level was further demonstrated by the preparation of emollient esters such as myristyl myristate reported by Evonik researchers in collaboration with the group of Liese (Scheme 3). ${ }^{16}$ The esterification could be performed without solvent, mixing equimass amounts 


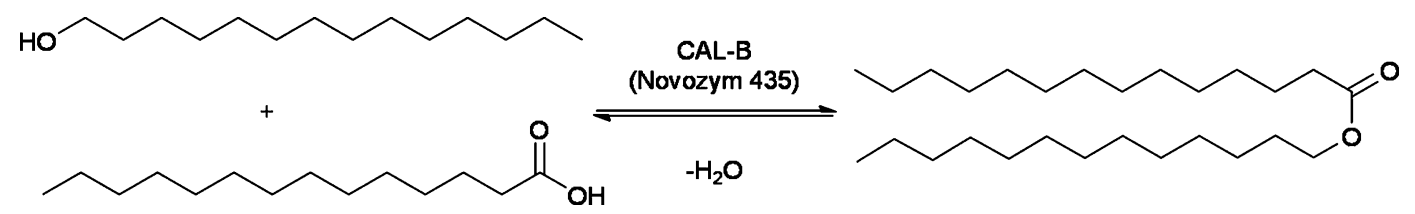

Scheme 3

of reactants at $75{ }^{\circ} \mathrm{C}$ in the presence of Novozym 435. An outstanding space time yield of $6731 \mathrm{~g} \mathrm{~d}^{-1} \mathrm{~L}^{-1}$ was achieved for myristyl myristate.

As summarized in the SWOT analysis (Fig. 2), carboxylic acids are more benign and environmentally friendly than other common acyl donors (e.g., trifluoroesters, vinyl acetate),${ }^{17}$ as they are characterized by a lower flammability and explosion hazard, and do not form harmful waste. They show a good compliance to the 1st, 2nd, 3rd, and 10th principle of Green Chemistry, and should therefore be preferred to activated (and more toxic) acyl donors whenever possible. On the other hand, carboxylic acids suffer often from a limited solubility; therefore, the condensation with alcohols might require the use of an excess of alcohol or of a solvent.

\section{Carboxylic acids}

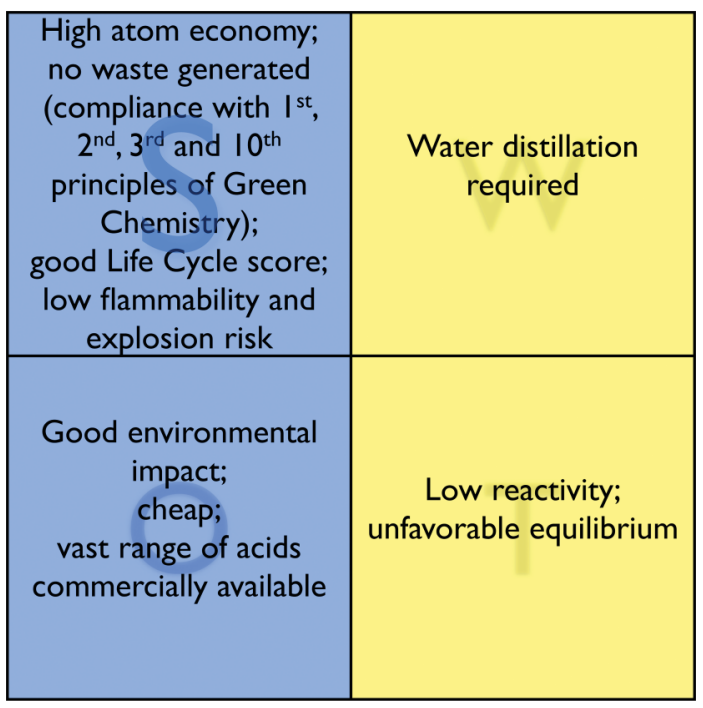

Fig. 2 SWOT analysis for carboxylic acids.

\section{Trihaloesters}

2,2,2-Trichloroethyl groups were introduced as activated acyl donors in the early 1990s in the first acylation studies in organic solvents. ${ }^{18}$ The activation is poor $^{8}$ and transesterifications are therefore slow. As a consequence, only limited examples (none in the last five years) can be found in literature where this leaving group is used.

2,2,2-Trifluoroethyl esters $\mathbf{2 b}$ are slightly more activated than their trichloro counterparts, but are still significantly less used than vinyl esters. ${ }^{19,20}$ Their synthesis is easily accomplished either via carbodiimide coupling of acids and alcohols ${ }^{21,22}$ or using acid chlorides and the alcohol. ${ }^{19,23-25}$
A few years ago, the dynamic kinetic resolution of a series of allylic alcohols using 2,2,2-trifluoroethyl butanoate in the presence of Subtilisin-CLEC and a Ru complex as the racemization catalyst was reported. ${ }^{20}$ For the example reported in Scheme 4, an atom economy of $68.6 \%$ and an E-factor of 11.8 were obtained. Overall, the process is quite poor.

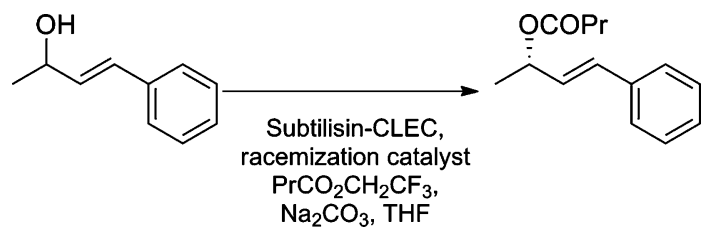

Scheme 4

The byproduct (trifluoroethanol) gets a relatively good score in the LCA (life cycle assessment) and has a low reactivity, but raises serious issues from the health and waste points of view (Fig. 3), as pointed out in a recent assessment of common organic solvents. ${ }^{17}$ Indeed, it is a harmful and non-biodegradable substance, which is against what the relevant principles of Green Chemistry advise. Therefore, its use and generation should be avoided, also in consideration of possible future health related regulations.

\section{Trihaloesters}

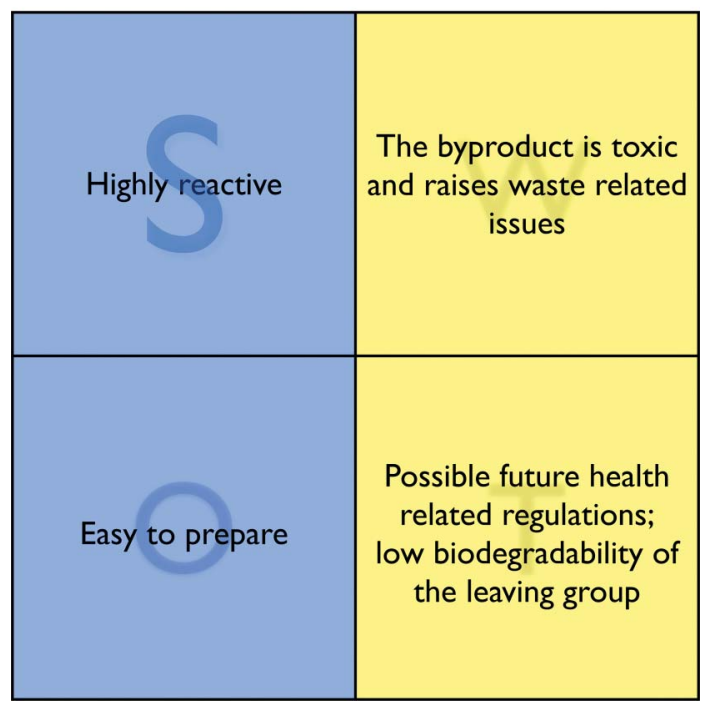

Fig. 3 SWOT analysis for trihaloesters.

\section{Anhydrides}

Although acyclic anhydrides have been applied early in enzymatic reactions, ${ }^{26}$ they find moderate application in 
enzyme-mediated ester synthesis (see Table 1). Their ability to acylate the enzyme, deactivating it, as well as the competing, unselective background reaction, and the occurrence of undesired side reactions related to the acid released account for their limited use. ${ }^{9-11}$ Moreover, anhydrides are irritating. The SWOT table summarizes all these aspects.

Nonetheless, cyclic anhydrides (e.g., succinic anhydride) are more often used for the kinetic resolution of alcohols than their acyclic equivalent, even on an industrial scale. ${ }^{27}$ Indeed, they offer the advantage of an easy separation of the product monoester (through extraction with bases) from the unreacted alcohol. Moreover, acylations with cyclic anhydrides have an atom economy of $100 \%$, although it must be taken into account that the hemiester obtained is normally not the desired final product, and an additional step (the hydrolysis) has to be performed.

One such example ${ }^{28}$ (Scheme 5) is given by the resolution of $(R, S)$ - $N$-(tert-butoxycarbonyl)-3-hydroxymethylpiperidine with succinic anhydride and BCL.

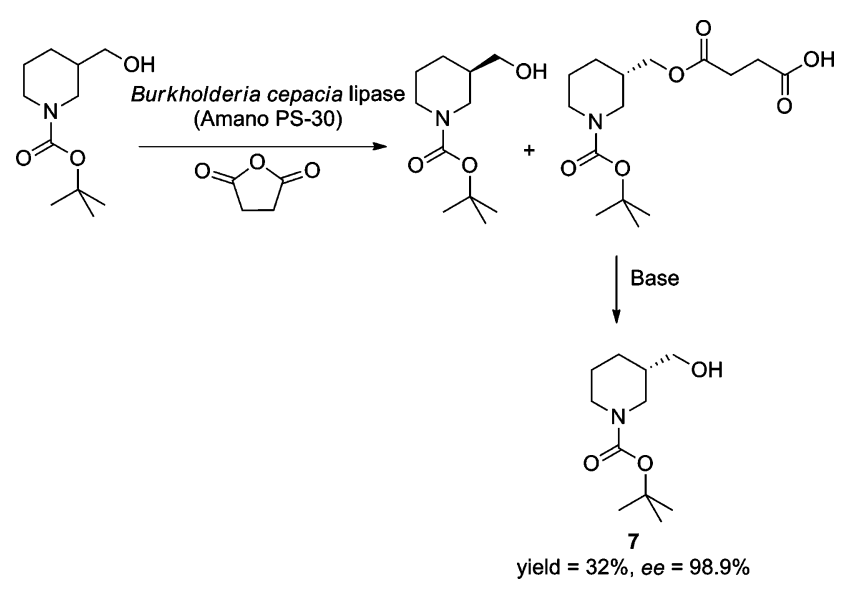

Scheme 5

The $(S)$-hemisuccinic ester was easily separated and hydrolyzed to give 7, intermediate in the preparation of a tryptase inhibitor. Reiteration of the process allowed 7 to be obtained in $32 \%$ yield and $98.9 \%$ ee. A calculation of the E-factor for the first cycle only gives a value of 15 , which makes the process quite poor.

In conclusion, cyclic anhydrides show a very good agreement with the 1st, 2nd, 3rd and 10th principle of Green Chemistry, and appear therefore as relatively green acyl donors (Fig. 4). On the other hand, acyclic anhydrides perform worse from the atom economy and waste generation standpoint, and are therefore a less convenient choice.

\section{Enol esters}

By far the best activated and most used acyl donors on a small scale (see Table 1) are enol esters such as vinyl acetate (VA, $\mathbf{4 a}$, $\mathrm{R}=\mathrm{H}$ ), isopropenyl acetate (IPA, $\mathbf{4 b} \mathrm{R}=\mathrm{Me}$ ) and ethoxyvinyl esters $(\mathbf{4 c}, \mathrm{R}=\mathrm{OMe}){ }^{29-31}$ The leaving group is an enol that immediately tautomerizes to the ketoform (Scheme 6). Thus, no nucleophile remains and the reaction becomes irreversible. Some vinyl esters and IPA are commercially available, since they are

\section{Anhydrides}

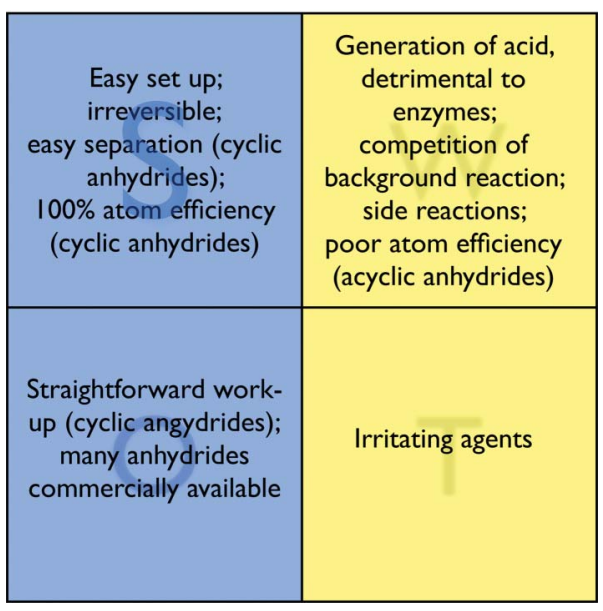

Fig. 4 SWOT analysis for anhydrides.

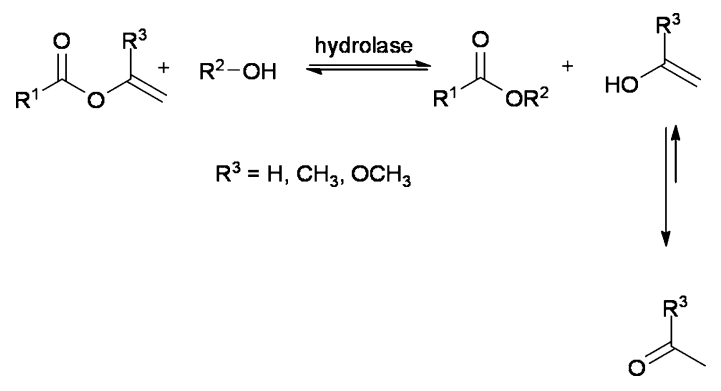

Scheme 6

building blocks in polymer chemistry and are used as acylating agents in non-enzymatic reactions as well. Pd- or acid catalyzed transesterifications ensure the conversion of these bulk chemicals into the desired vinyl or isopropenyl esters. ${ }^{11}$ Alkoxyvinyl esters can also be synthesized from the corresponding acid and acetylene using different Ru catalysts. ${ }^{32,33}$ These acyl donors (especially the cheap VA) are often used in large excess, thus making the use of a solvent unnecessary.

When comparing their SWOT analyses, VA emerges as the cheapest and the most reactive, but these advantages are severely undermined by a serious drawback, i.e. the generation of a stoichiometric amount of acetaldehyde. Not only can acetaldehyde deactivate some lipases (CRL and GCL) by formation of imine bonds with the lysine residues, it also presents major process obstacles, ${ }^{34}$ such as the low flash $\left(-40{ }^{\circ} \mathrm{C}\right)$ and boiling $\left(2{ }^{\circ} \mathrm{C}\right)$ points and the explosive properties of airacetaldehyde mixtures. ${ }^{35}$ Moreover, acetaldehyde is an irritating agent ${ }^{36}$ that has been classified as an inhalation carcinogen ${ }^{37}$ $\left(\mathrm{LD}_{50}=661 \mathrm{mg} \mathrm{kg}^{-1}\right)$ a few years ago. Another safety hazard is represented by the potential of VA to undergo exothermic polymerization in the gas phase. ${ }^{38}$ This risk should be taken into account whenever VA is used in large excess and removed by distillation at the end of the reaction, as is the case, among others, in the synthesis of more hindered vinyl esters donors. For all these reasons, only very few reports on the scale-up of vinyl esters mediated acylations can be found in the literature. ${ }^{39,40}$ In one such example from Roche (Scheme 7) ${ }^{40} \mathrm{VA}$ is used for 


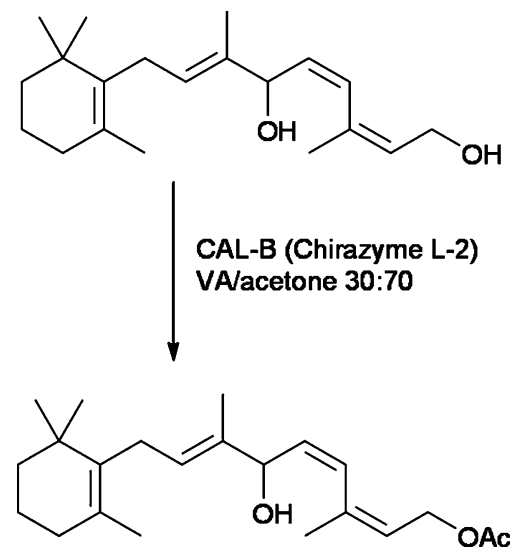

Scheme 7

the Chirazyme L-2 mediated acetylation of an intermediate for Vitamin A synthesis. At 100\% conversion, $1.6 \mathrm{~kg}$ of desired product are prepared per day, corresponding to an acceptable Efactor of 8.12. The addition of EDTA and of ppm of an organic base and an antioxidant to the reaction mixture to protect the enzyme against degradation could not be taken into account in the calculation.

When using IPA or ethoxyvinyl acetate, ${ }^{41}$ the byproducts are acetone and ethyl acetate, respectively. Since both compounds are unreactive towards lysine, ${ }^{9}$ no enzyme deactivation is observed. Moreover, they are significantly more benign than acetaldehyde. ${ }^{36}$ However, IPA and ethoxyvinyl acetate suffer from some of the same disadvantages (hazardous traditional synthesis, polymerization issue) as VA, and no scale up reaction have been reported so far. Although a relatively mild synthesis for alkoxyvinyl esters was proposed in $1993,{ }^{33}$ no application on a large scale are known, probably due to the cost of the $\mathrm{Ru}$ catalyst.

The atom economy of acylations with enol esters is generally poor $(50-60 \%)$ when small alcohols are used (Fig. 5-7), but improves when increasing the molecular weight of the acyl acceptor. Obviously, it is higher for VA than for IPA and ethoxyvinyl esters.

\section{Vinyl acetate}

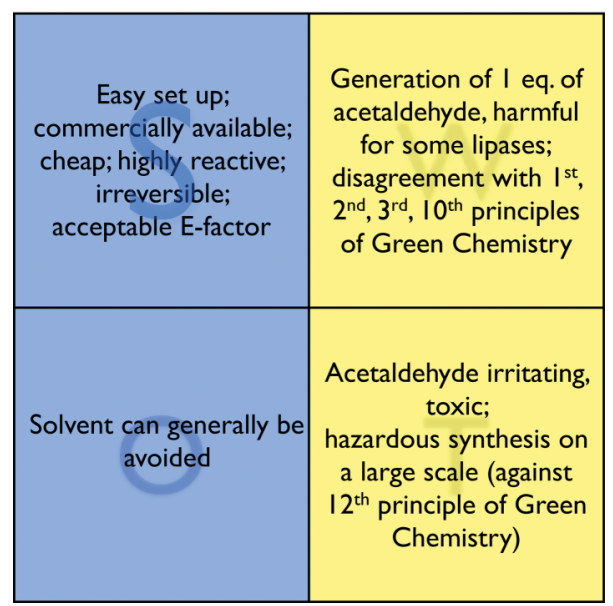

Fig. 5 SWOT analysis for vinyl acetate.

\section{Isopropenyl acetate}

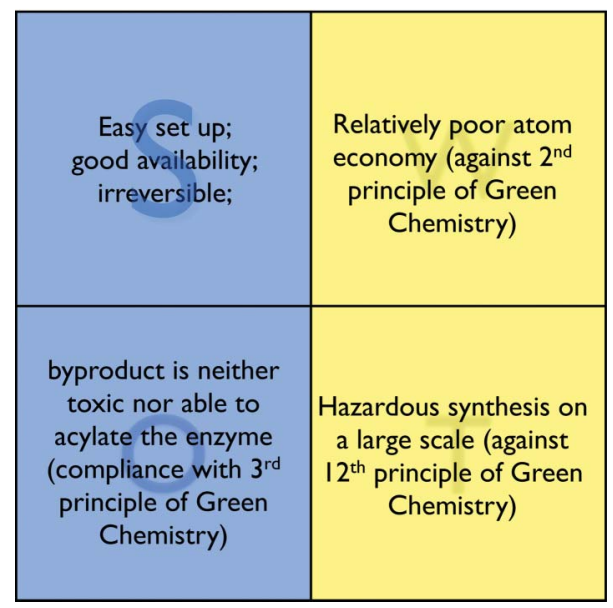

Fig. 6 SWOT analysis for isopropenyl acetate.

\section{Ethoxyvinyl acetate}

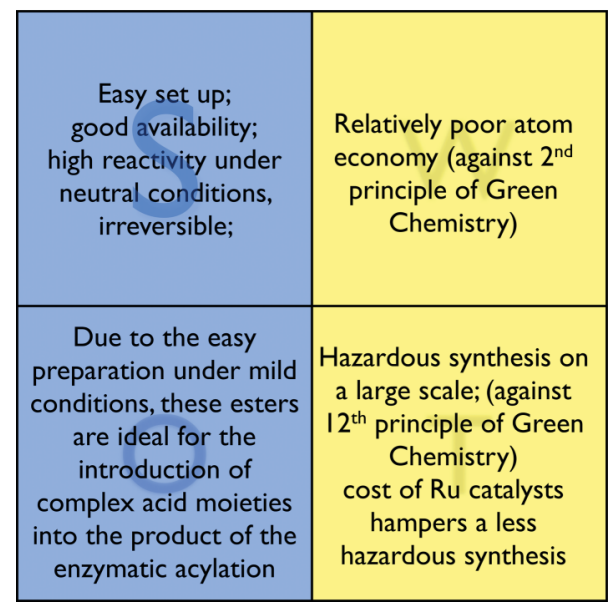

Fig. 7 SWOT analysis for ethoxyvinyl esters.

\section{Alkyl methoxyacetates}

Ethylmethoxyacetate was introduced by BASF researchers in the $1990 \mathrm{~s}^{42}$ for the enzymatic resolution of chiral amines. Since then, esters of methoxyacetic acid have been playing an important role in the large-scale production of optically pure aliphatic and benzyl amines, and amino alcohols. ${ }^{27,43-45}$ The methoxy substituent remarkably enhances the carbonyl reactivity, so that the initial acylation rate for ethylmethoxyacetate is 100 times faster than the corresponding reaction with, for example, ethyl butyrate. ${ }^{46}$ Additionally, these acyl donors are unrivaled in terms of selectivity, which is especially increased when using esters of secondary alcohols like isopropyl methoxyacetate. ${ }^{44}$

In a typical procedure (Scheme 8), ${ }^{42}$ equimolar amounts (165 mmol) of 1-phenylethylamine and ethylmethoxy acetate are converted in MTBE in the presence of $2 \mathrm{~g}$ of Burkholderia plantarii lipase. At 52\% conversion, the reaction affords $48 \%$ of $(R)$-amide in $93 \%$ ee. The procedure is characterized by a quite high atom economy $(80.7 \%)$, and an E-factor as high as 11.1 (Fig. 8). 
<smiles>CCOC(=O)COC</smiles>

Methoxyacetates

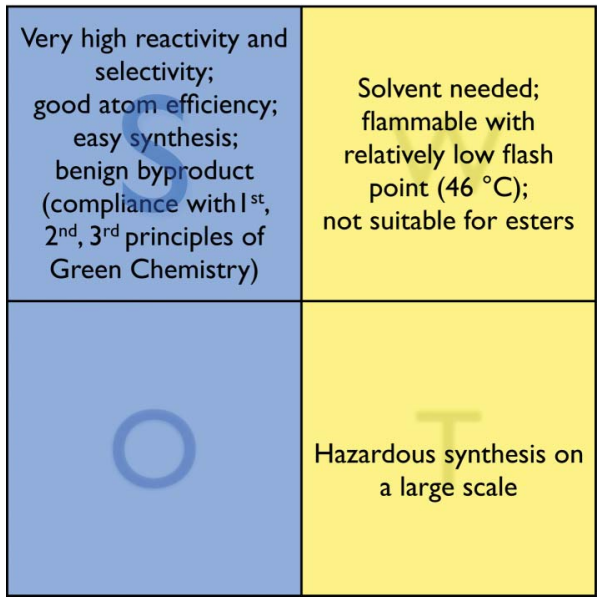

Fig. 8 SWOT analysis for alkyl methoxyacetates.

\section{Oxime esters}

Oxime esters, first reported in $1989,{ }^{47}$ have scarcely been used in the last 5 years. They are generally considered irreversible acyl donors, even though a reversible behaviour has been sometimes reported. ${ }^{10}$ Besides the simple acetone oxime, ${ }^{48,49}$ more complex oxime esters have been recently proposed ${ }^{34}$ and proved even more successful. Their preparation involves the DCC coupling of oximes and acids or the condensation of oximes and acid chlorides. ${ }^{11}$

An interesting example of industrial application of an oxime ester ${ }^{50}$ is the acylation of the primary alcohol of Ribavirin with Cbz-protected L-alanyl oxime ester in the presence of CALB (Chirazyme) applied by Schering-Plough on a pilot scale (Scheme 9).

The acyl donor was prepared in situ by coupling acetone oxime and Cbz-Ala in the presence of di-tert-butyl dicarbonate in THF, and directly reacted with ribavirin. The atom economy for the esterification is $86.1 \%$, but the $\mathrm{E}$ factor for the overall process is as high as 33.2, which reflects the generation of a large amount of waste (Fig. 9).<smiles>CC(C)=NOC(=O)[C@H](C)NC(=O)OCc1ccccc1</smiles>

Ribavirin

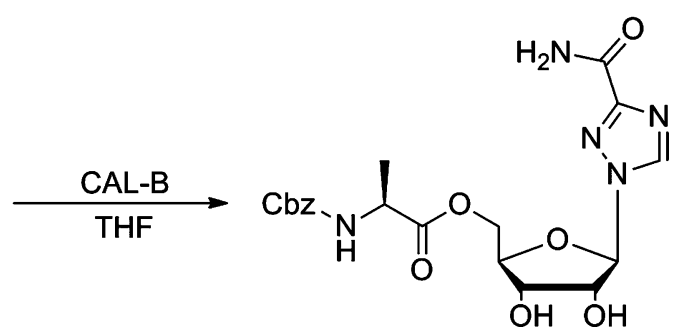

Scheme 9

Oxime esters

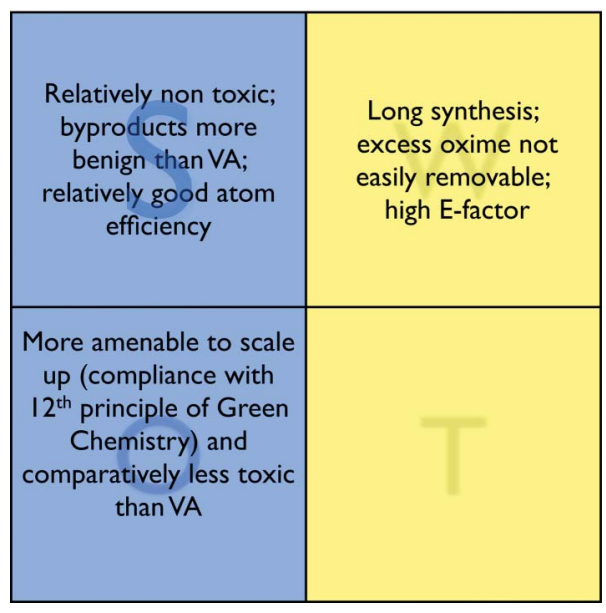

Fig. 9 SWOT analysis for oxime esters.

\section{Conclusions}

When performing an enzymatic esterification on a large scale, the simple carboxylic acid appears still as the best acyl donor from the Green Chemistry perspective. It has a very good atom economy, generates water as the only waste and shows a relatively good environmental impact. Besides, a very broad range of acids are readily available, which can be employed directly. However, this advantage is counterbalanced by the necessity to continuously remove the water formed in order to shift the unfavourable equilibrium.

On a small scale, the use of an activated donor rather than the simple acid is still preferred, as witnessed by the current literature (Table 1). However, when assessing all the reagents available for their "greenness", the popularity of vinyl acetate (and, to a lesser extent, of enol esters in general) seems largely undeserved. VA does not comply with any of the relevant Green Chemistry principles considered here. The safety issues related to its synthesis and, above all, the generation of the 
environmentally unfriendly and harmful acetaldehyde, in our opinion, call for the search for more sustainable alternatives. Equally serious drawbacks from a green perspective affect the use of trihaloesters.

Alkyl methoxyacetates emerge as a very good choice for the acylation of amines. The high reactivity and selectivity are accompanied by a satisfying atom economy, a relatively easy synthesis, and the formation of simple alcohols as byproducts. Unfortunately, the unfavourable equilibrium makes alkyl methoxyacetates inapplicable to the esterification of alcohols. In this case, cyclic anhydrides should be preferred whenever possible, as they are characterized by $100 \%$ atom economy and simplify the separation of the desired product from the unreacted alcohol. In this respect, they offer more advantages than oxime esters, too. Indeed, the latter are also characterized by a good atom economy and by the formation of relatively benign byproducts, but suffer from a laborious synthesis and the difficult separation of the oxime that is used in excess.

Overall, the analysis performed here confirms that to define any enzymatic process as green without evaluating the actual impact of the reagents involved is misleading. As demonstrated here, greener alternatives to the frequently used enol esters are available. In particular, carboxylic acids represent a much more sustainable choice, provided that an efficient water removal protocol is applied. Clearly the key to implementing a truly green process resides not only in the underlying chemistry, but also in the process engineering. The combination of both fields is a powerful tool opening up novel opportunities towards Green Chemistry.

\section{Acknowledgements}

Dr V. R. Calderone is gratefully acknowledged for inspiring the graphical part of this article.

\section{Notes and references}

1 W. D. Fessner and T. Anthonsen, Modern Biocatalysis: stereoselective and environmentally friendly reactions, Wiley- $\mathrm{VCH}$, Weinheim, 2009.

2 A. Liese, K. Seelback and C. Wandrey, Industrial Biotransformations, Wiley-VCH, Weinheim, 2006.

3 P. T. Anastas and J. C. Warner, Green Chemistry: Theory and Practice, Oxford University Press, New York, 1998.

4 P. T. Anastas and J. B. Zimmerman, Environ. Sci. Technol., 2003, 37, 94A-101A.

5 P. T. Anastas and M. M. Kirchhoff, Acc. Chem. Res., 2002, 35, 686693.

6 R. A. Sheldon, I. W. C. E. Arends and U. Hanefeld, Green Chemistry and Catalysis, Wiley-VCH, Weinheim, 2007.

7 U. T. Bornscheuer and R. J. Kazlauskas, Hydrolases in Organic Synthesis, Wiley-VCH, Weinheim, New York, Chichester, Brisbane, Singapore, Toronto, 2005.

8 H. Stecher and K. Faber, Synthesis, 1997, 1-16.

9 R. Chênevert, N. Pelchat and F. Jacques, Curr. Org. Chem., 2006, 10, 1067-1094.

10 K. Faber and S. Riva, Synthesis, 1992, 895-910.

11 U. Hanefeld, Org. Biomol. Chem., 2003, 1, 2405-2415.

12 B. M. Trost, Science, 1991, 254, 1471-1477.

13 R. A. Sheldon, Green Chem., 2007, 9, 1273-1283.
14 R. C. Appleby, Modern Business Administration, Pearson Education Ltd, 1994.

15 C. Korupp, R. Weberskirch, J. J. Muller, A. Liese and L. Hilterhaus, Org. Process Res. Dev., 2010, 14, 1118-1124.

16 L. Hilterhaus, O. Thum and A. Liese, Org. Process Res. Dev., 2008, 12, 618-625.

17 R. K. Henderson, C. Jimenez-Gonzalez, D. J. C. Constable, S. R. Alston, G. G. A. Inglis, G. Fisher, J. Sherwood, S. P. Binks and A. D. Curzons, Green Chem., 2011, 13, 854-862.

18 A. M. Klibanov, Acc. Chem. Res., 1990, 23, 114-120.

19 C. M. Monteiro, N. M. T. Lourenço and C. A. M. Afonso, Tetrahedron: Asymmetry, 2010, 21, 952-956.

20 M.-J. Kim, H. K. Lee and J. Park, Bull. Korean Chem. Soc., 2007, 28, 2096-2098.

21 N. Baba, M. K. Alam, Y. Mori, S. S. Haider, M. Tanaka, S. Nakajima and S. Shimizu, J. Chem. Soc., Perkin Trans. 1, 2001, 221-223.

22 T. Miyazawa, S. Nakajo, M. Nishikawa, K. Hamahara, K. Imagawa, E. Ensatsu, R. Yanagihara and T. Yamada, J. Chem. Soc., Perkin Trans. 1, 2001, 82-86.

23 V. K. Aggarwal, D. E. Jones and A. M. Martin-Castro, Eur. J. Org. Chem., 2000, 2939-2945.

24 S. Gedey, A. Liljeblad, L. Lázár, F. Fülöp and L. T. Kanerva, Tetrahedron: Asymmetry, 2001, 12, 105-110.

25 X. G. Li and L. T. Kanerva, Org. Lett., 2006, 8, 5593-5596.

26 D. Bianchi, P. Cesti and E. Battistel, J. Org. Chem., 1988, 53, 55315534.

27 A. Schmid, J. S. Dordick, B. Hauer, A. Kiener, M. Wubbolts and B. Witholt, Nature, 2001, 409, 258-268.

28 A. Goswami, J. M. Howell, E. Y. Hua, K. D. Mirfakhrae, M. C. Soumeillant, S. Swaminathan, X. H. Qian, F. A. Quiroz, T. C. Vu, X. B. Wang, B. Zheng, D. R. Kronenthal and R. N. Patel, Org. Process Res. Dev., 2001, 5, 415-420.

29 M. Degueil-Castaing, B. De Jeso, S. Drouillard and B. Maillard, Tetrahedron Lett., 1987, 28, 953-954.

30 Y. F. Wang, J. J. Lalonde, M. Momongan, D. E. Bergbreiter and C. H. Wong, J. Am. Chem. Soc., 1988, 110, 7200-7205.

31 S. Raucher and B. L. Bray, J. Org. Chem., 1987, 52, 2332-2333.

32 T. Mitsudo, Y. Hori, Y. Yamakawa and Y. Watanabe, Tetrahedron Lett., 1986, 27, 2125-2126.

33 Y. Kita and S. Akai, Chem. Rec., 2004, 4, 363-372.

34 T. Storz, J. Gu, B. Wilk and E. Olsen, Tetrahedron Lett., 2010, 51, $5511-5515$.

35 L. G. Britton, American Institute of Chemical Engineers, 1998, pp. $138-148$.

36 http://chemiekaarten.sdu.nl/chkonline/.

37 R. Gomes and M. E. Meek, J. Environ. Sci. Health, Part C: Environ. Carcinog. Ecotoxicol. Rev., 2001, 19, 1-21.

38 H. Reiss and M. A. Chowdhury, J. Phys. Chem., 1984, 88, 6667-6670.

39 B. Orsat, B. Wirz and S. Bischof, Chimia, 1999, 53, 579-584.

40 W. Bonrath, R. Karge and T. Netscher, J. Mol. Catal. B: Enzym., 2002, 19-20, 67-72.

41 D. Klomp, K. Djanashvili, N. C. Svennum, N. Chantapariyavat, C.-S. Wong, F. Vilela, T. Maschmeyer, J. A. Peters and U. Hanefeld, Org. Biomol. Chem., 2005, 3, 483-489.

42 F. Balkenhohl, K. Ditrich, B. Hauer and W. Ladner, J. Prakt. Chem. 1997, 339, 381-384.

43 M. Höhne and U. T. Bornscheuer, Chem CatChem, 2009, 1, 42-51.

44 K. Ditrich, Synthesis, 2008, 2283-2287.

45 M. Breuer, K. Ditrich, T. Habicher, B. Hauer, M. Keßeler, R. Stürmer and T. Zelinski, Angew. Chem., Int. Ed., 2004, 43, 788-824.

46 M. Cammenberg, K. Hult and S. Park, ChemBioChem, 2006, 7, $1745-1749$.

47 A. Ghogare and G. S. Kumar, J. Chem. Soc., Chem. Commun., 1989, 1533-1535.

48 R. Pulido and V. Gotor, Carbohydr. Res., 1994, 252, 55-68.

49 F. Moris and V. Gotor, J. Org. Chem., 1993, 58, 653-660.

50 M. Tamarez, B. Morgan, G. S. K. Wong, W. Tong, F. Bennett, R. Lovey, J. L. McCormick and A. Zaks, Org. Process Res. Dev., 2003, 7, 951-953. 